\title{
Stachybotrysin, an Osteoclast Differentiation Inhibitor from the Marine- derived Fungus Stachybotrys sp. KCB13F013
}

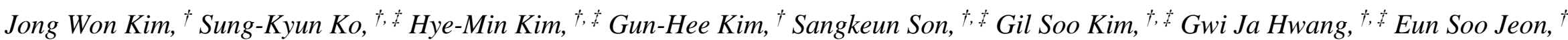
Kee-Sun Shin, ${ }^{\S}$ In-Ja Ryoo, ${ }^{\dagger}$ Young-Soo Hong, ${ }^{\dagger}, *$ Hyuncheol Oh, ${ }^{\perp}$ Kyung Ho Lee, ${ }^{\dagger}$ Nak-Kyun Soung, ${ }^{\dagger},+$ Daisuke Hashizume, $"$ Toshihiko Nogawa, ${ }^{\nabla}$ Shunji Takahashi, ${ }^{\nabla, O}$ Bo Yeon Kim, ${ }^{\dagger}{ }^{\dagger}$ Hiroyuki Osada, ${ }^{\nabla}$ Jae-Hyuk Jang, ${ }^{\dagger, *},{ }^{*}$ and Jong Seog Ahn ${ }^{\dagger, *}, *$

${ }^{\dagger}$ Anticancer Agent Research Center, Korea Research Institute of Bioscience and Biotechnology, Cheongju, Chungbuk 28116, South Korea

tDepartment of Biomolecular Science, University of Science and Technology, Daejeon 34113, South Korea

${ }^{\S}$ Industrial Bio-materials Research Center, Daejeon 34141, South Korea

${ }^{\perp}$ College of Pharmacy, Wonkwang University, Iksan 54538, South Korea

"Materials Characterization Support Unit, RIKEN Center for Emergent Matter Science (CEMS), Wako, Saitama 351-0198, Japan

${ }^{\nabla}$ Chemical Biology Research Group, RIKEN CSRS, Wako, Saitama 351-0198, Japan

${ }^{\text {o }}$ RIKEN-KRIBB Joint Research Unit, RIKEN Global Research Cluster, Wako, Saitama 351-0198, Japan 


\section{Contents}

Fig. S1. ${ }^{1} \mathrm{H}$ NMR (800 MHz, DMSO-d6) spectrum of 1

Fig. S2. ${ }^{13} \mathrm{C}$ NMR (200 MHz, DMSO-d $)$ spectrum of 1

Fig. S3. COSY (DMSO-d $)$ spectrum of 1

Fig. S4. HMQC (DMSO-d6) spectrum of 1

Fig. S5. HMBC (DMSO-d6) spectrum of 1

Fig. S6. ROESY (DMSO-d $d_{6}$ ) spectrum of 1

Fig. S7. Positive HRESIMS spectrum of 1

Fig. S8. IR spectrum of 1

Fig. S9. ${ }^{1} \mathrm{H}$ NMR (700 MHz, DMSO-d6) spectrum of 2

Fig. S10. ${ }^{13} \mathrm{C}$ NMR (175 MHz, DMSO-d $)$ spectrum of 2

Fig. S11. COSY (DMSO-d $)_{6}$ spectrum of 2

Fig. S12. HSQC-DEPT (DMSO-d $)$ spectrum of 2

Fig. S13. HMBC (DMSO-d $)$ spectrum of 2

Fig. S14. ROESY (DMSO-d $)$ spectrum of 2

Fig. S15. ${ }^{1} \mathrm{H}$ NMR (700 MHz, Acetone- d6) spectrum of 2

Fig. S16. ${ }^{13} \mathrm{C}$ NMR (175 MHz, Acetone- d6) spectrum of 2 
Fig. S17. HSQC-DEPT (Acetone- $d_{6}$ ) spectrum of 2

Fig. S18. HMBC (Acetone- $d_{6}$ ) spectrum of 2

Fig. S19. ${ }^{1} \mathrm{H}$ NMR (700 MHz, DMSO- $\left.d_{6}\right)$ spectrum of methylation $2 \mathrm{c}$

Fig. S20. ${ }^{13} \mathrm{C}$ NMR (175 MHz, DMSO-d $)$ spectrum of methylation 2c

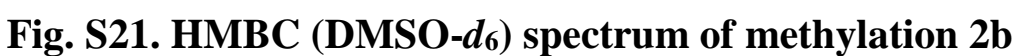

Fig. S22. ${ }^{1} \mathrm{H}$ NMR (900 MHz, DMSO-d6) spectrum of (R)-MTPA ester 2c

Fig. S23. ${ }^{1}$ H NMR (900 MHz, DMSO- $\left.d_{6}\right)$ spectrum of $(S)$-MTPA ester $2 d$

Fig. S24. Positive HRESIMS spectrum of 2

Fig. S25. IR spectrum of 2

Fig. S26. The difference of HMBC correlations (A) and NMR chemical shifts (B) in acetone- $d_{6}$ 


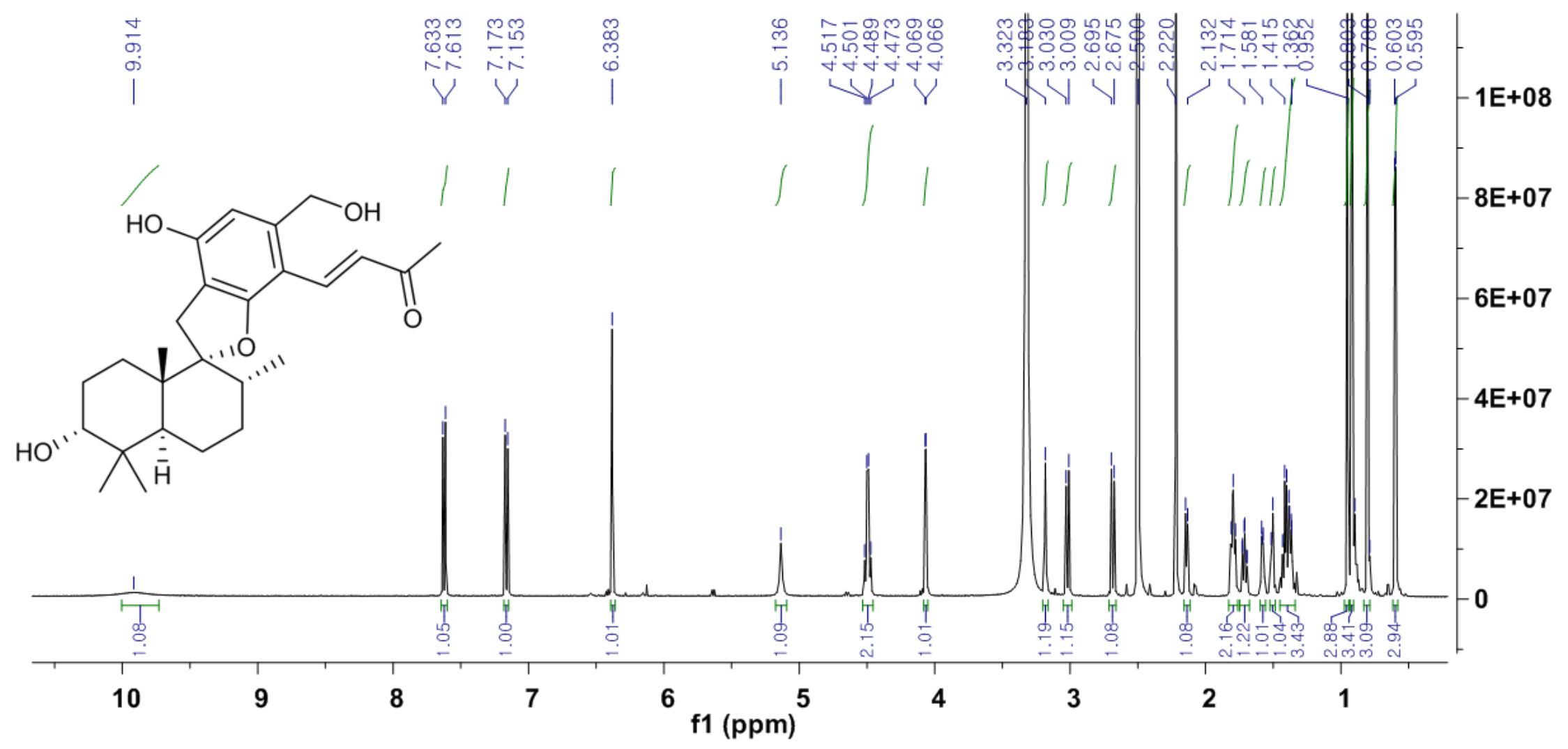

Fig. S1. ${ }^{1} \mathrm{H}$ NMR (800 MHz, DMSO- $\left.d_{6}\right)$ spectrum of 1 


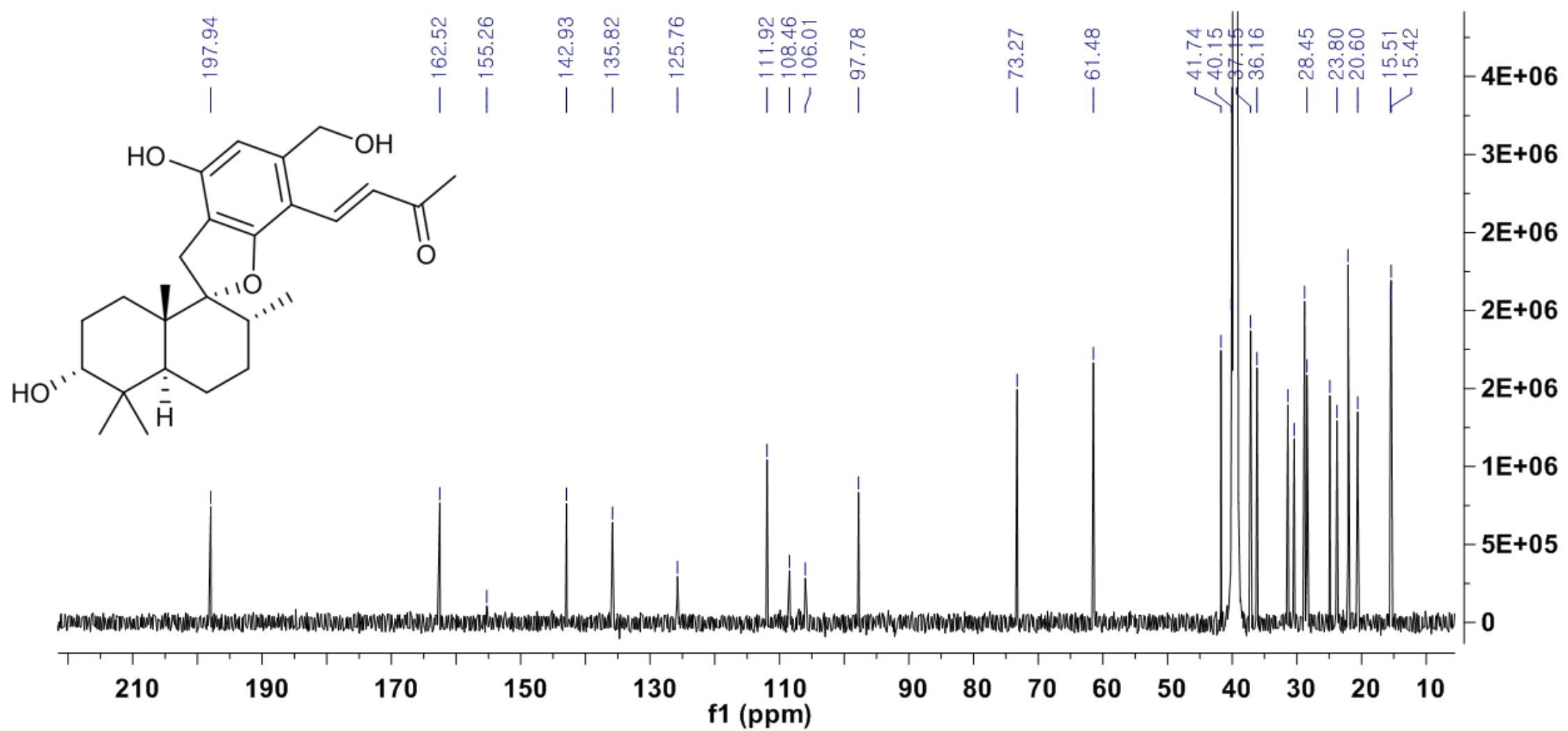

Fig. S2. ${ }^{13} \mathrm{C}$ NMR (200 MHz, DMSO-d6) spectrum of 1 


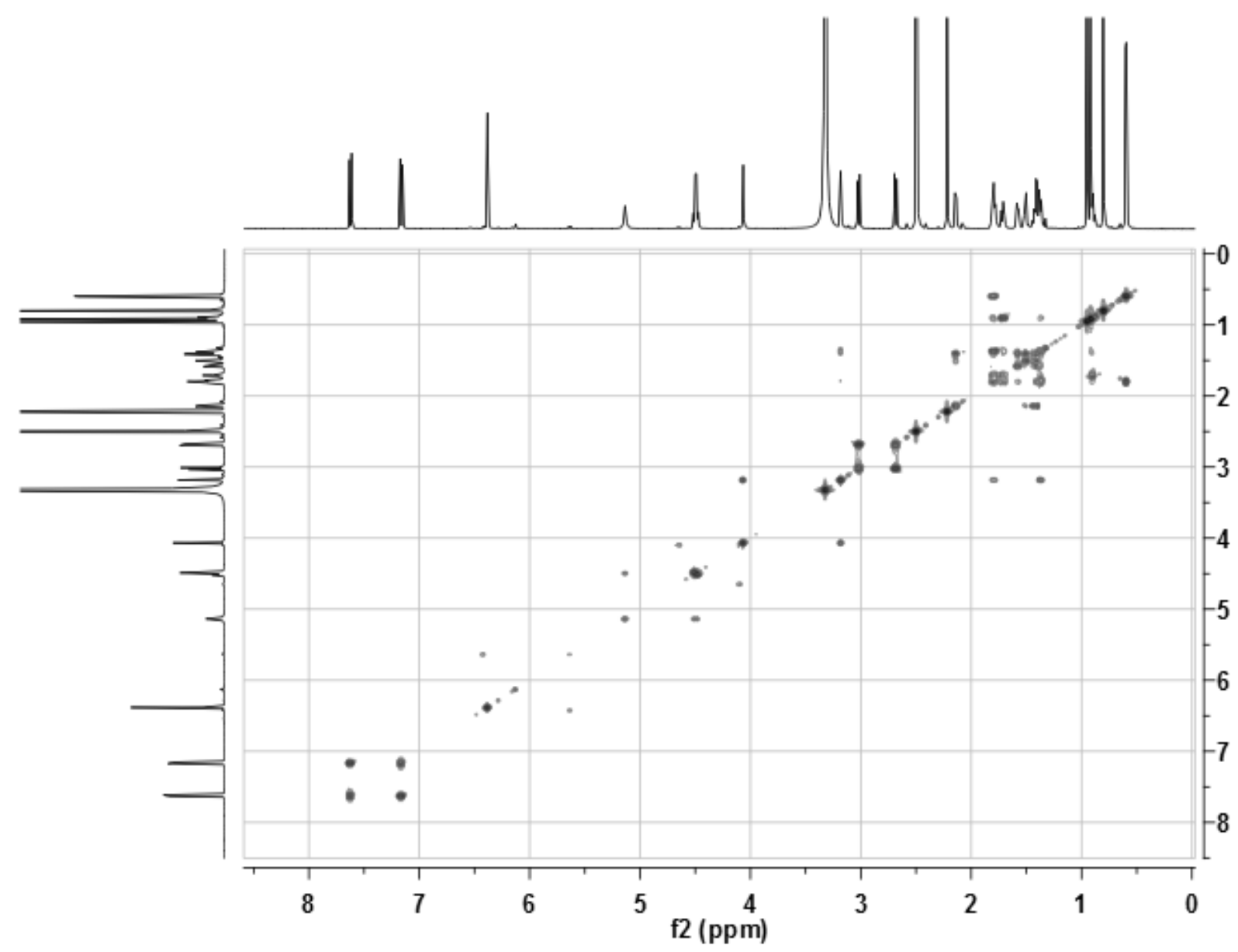

통

Fig. S3. COSY (DMSO-d $d_{\text {) }}$ spectrum of A 1 


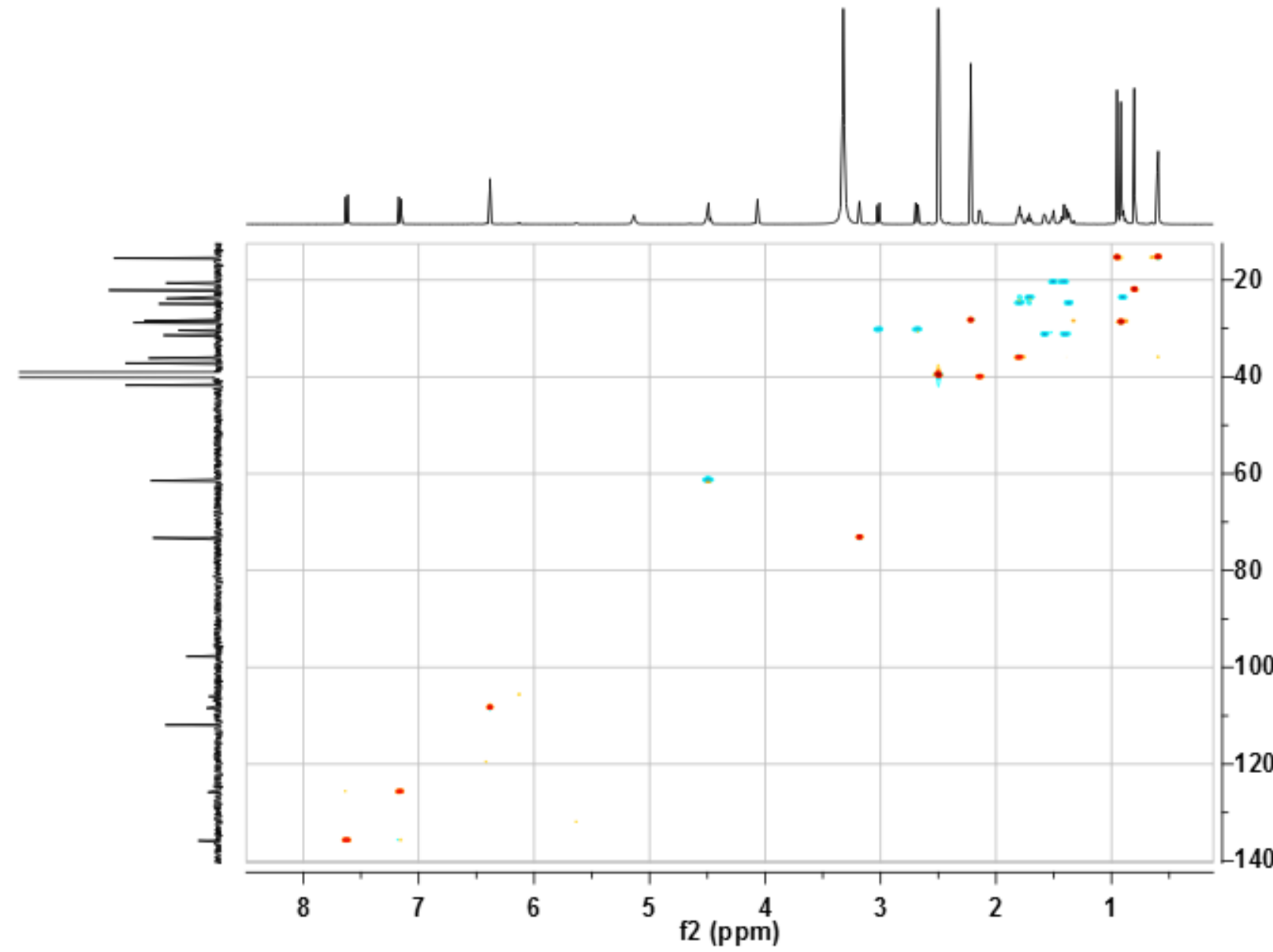

통

Fig. S4. HSQC-DEPT (DMSO-d $_{6}$ spectrum of A 1 


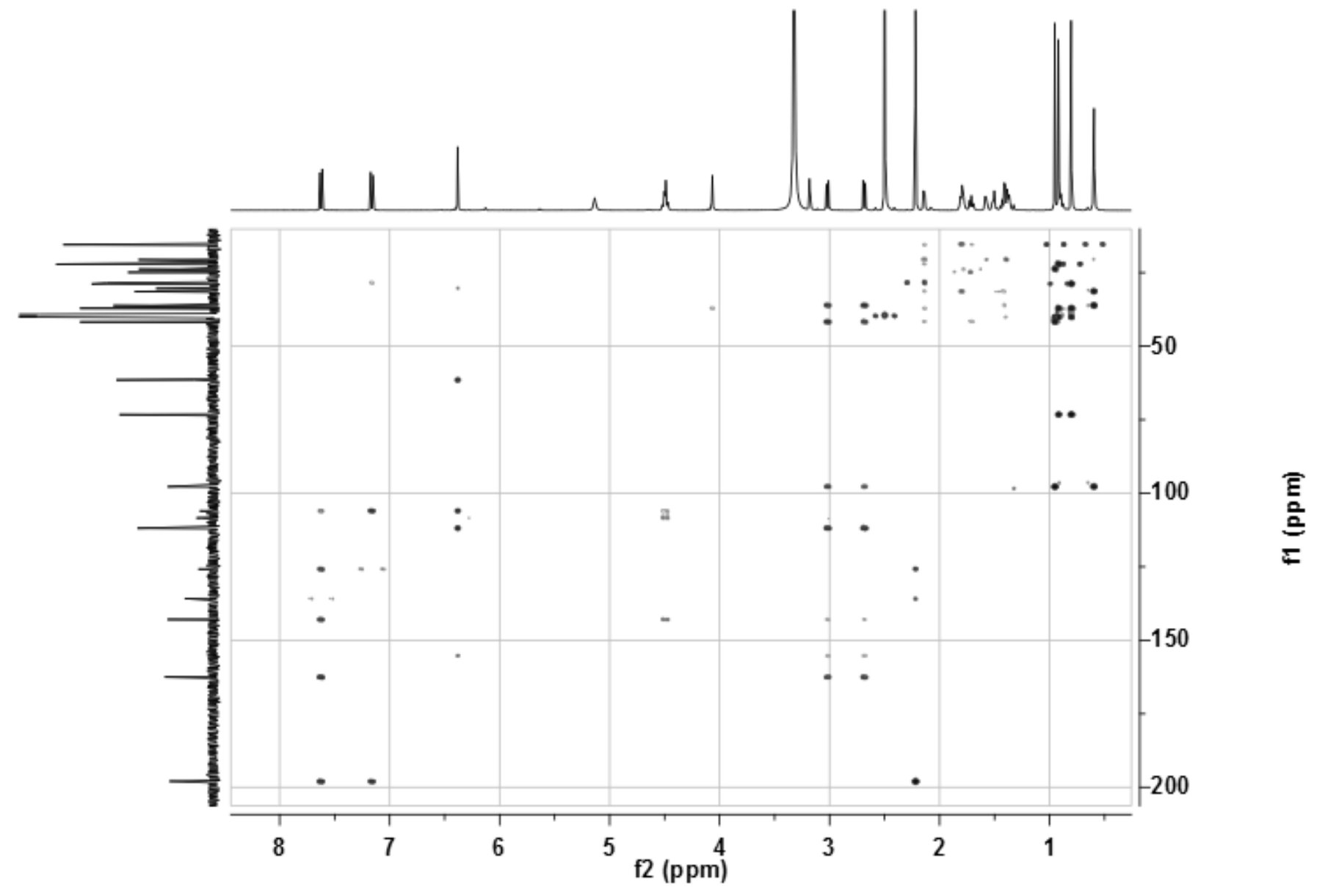

Fig. S5. HMBC (DMSO-d $d_{6}$ ) spectrum of 1 


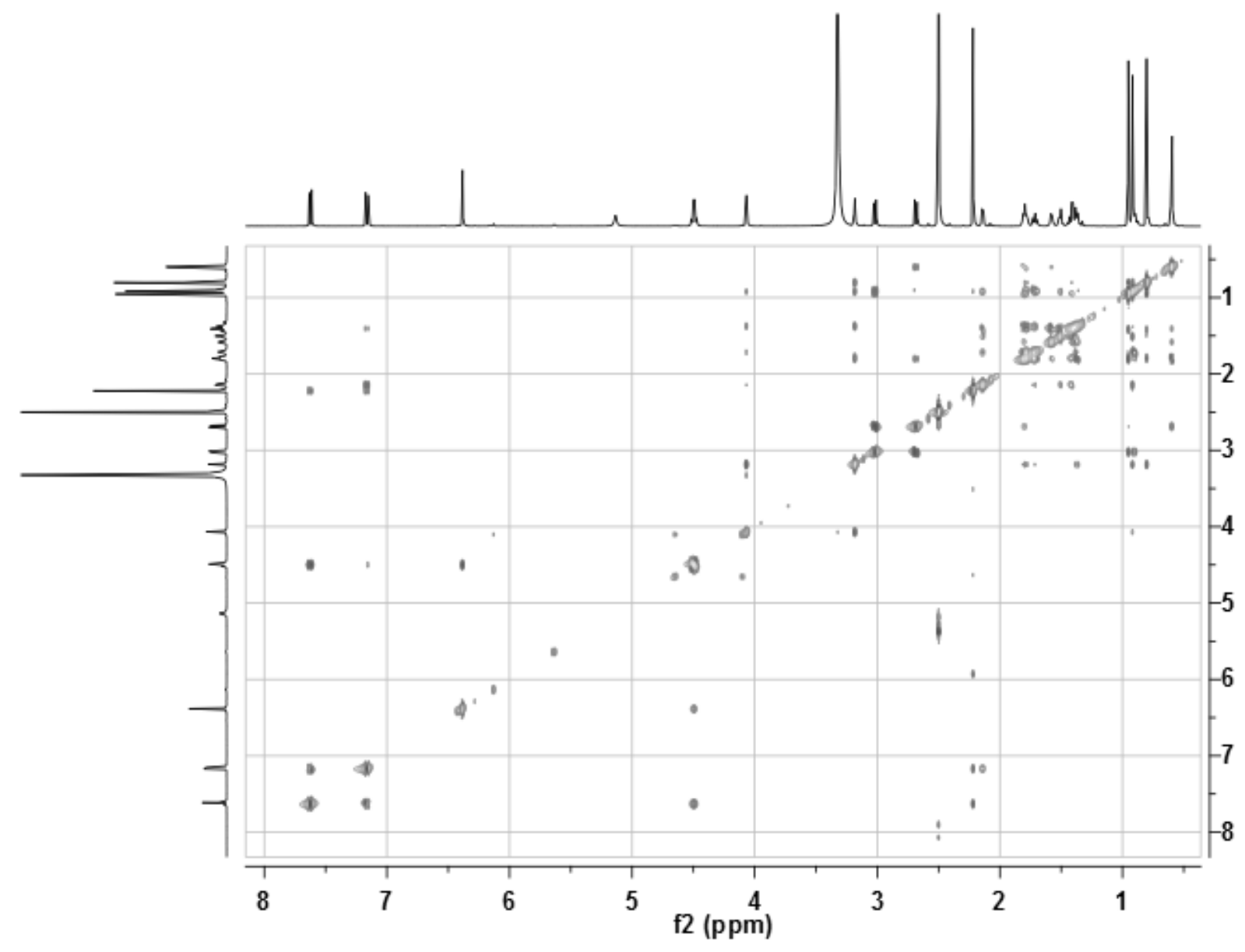

통

Fig. S6. ROESY (DMSO-d ${ }_{6}$ ) spectrum of 1 
Elemental Composition Report

Single Mass Analysis

Tolerance $=5.0$ PPM / DBE: $\min =-1.5, \max =50.0$

Element prediction: Off

Number of isotope peaks used for $\mathrm{i}-\mathrm{FIT}=3$

Monoisotopic Mass, Even Electron Ions

10 formula(e) evaluated with 1 results within limits (all results (up to 1000) for each mass)

Elements Used:

C: $1-30 \quad \mathrm{H}: 1-40 \quad$ O: $1-5 \quad \mathrm{Na}: 1-1$

Minimum:

Maximum:

5.0

Calc. Mass

451.2456

5.0

451.2460

$\begin{array}{lll}-0.4 & -0.9 & 8.5\end{array}$

5.0

$-1.5$

100

DBE

8.5

i-FIT

Norm

Conf(\%)

Formula

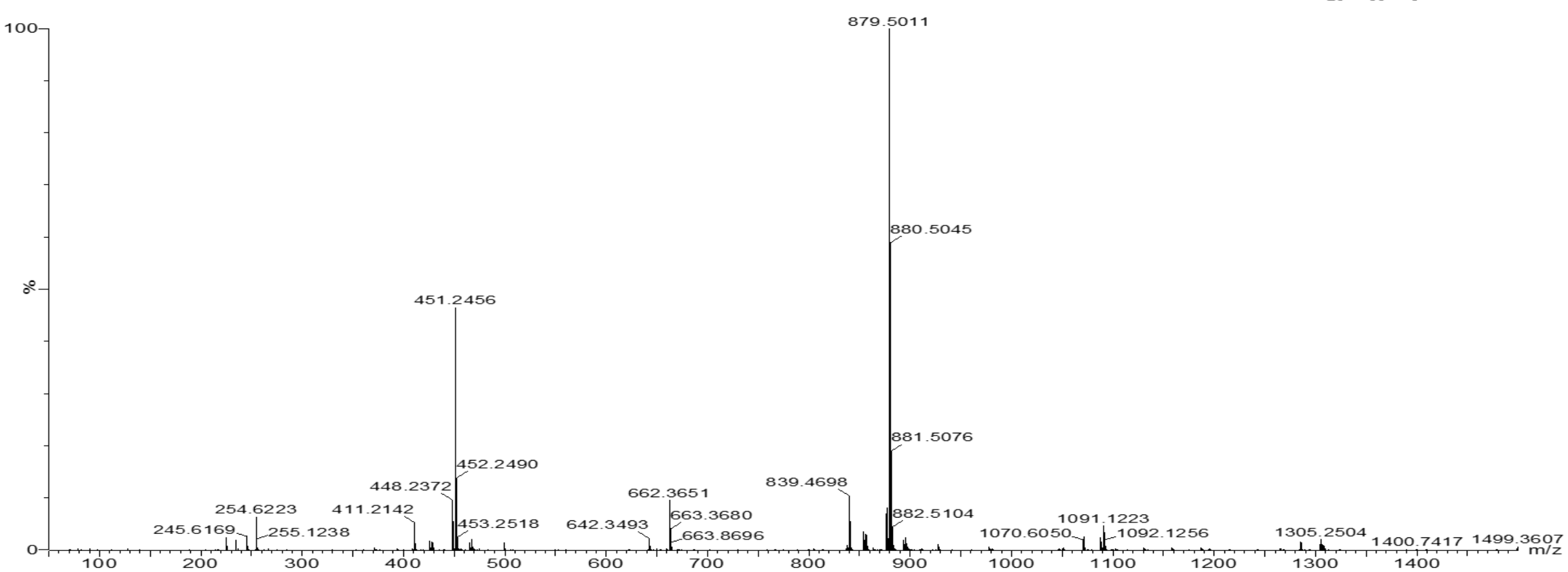

Fig. S7. Positive HRESIMS spectrum of 1 


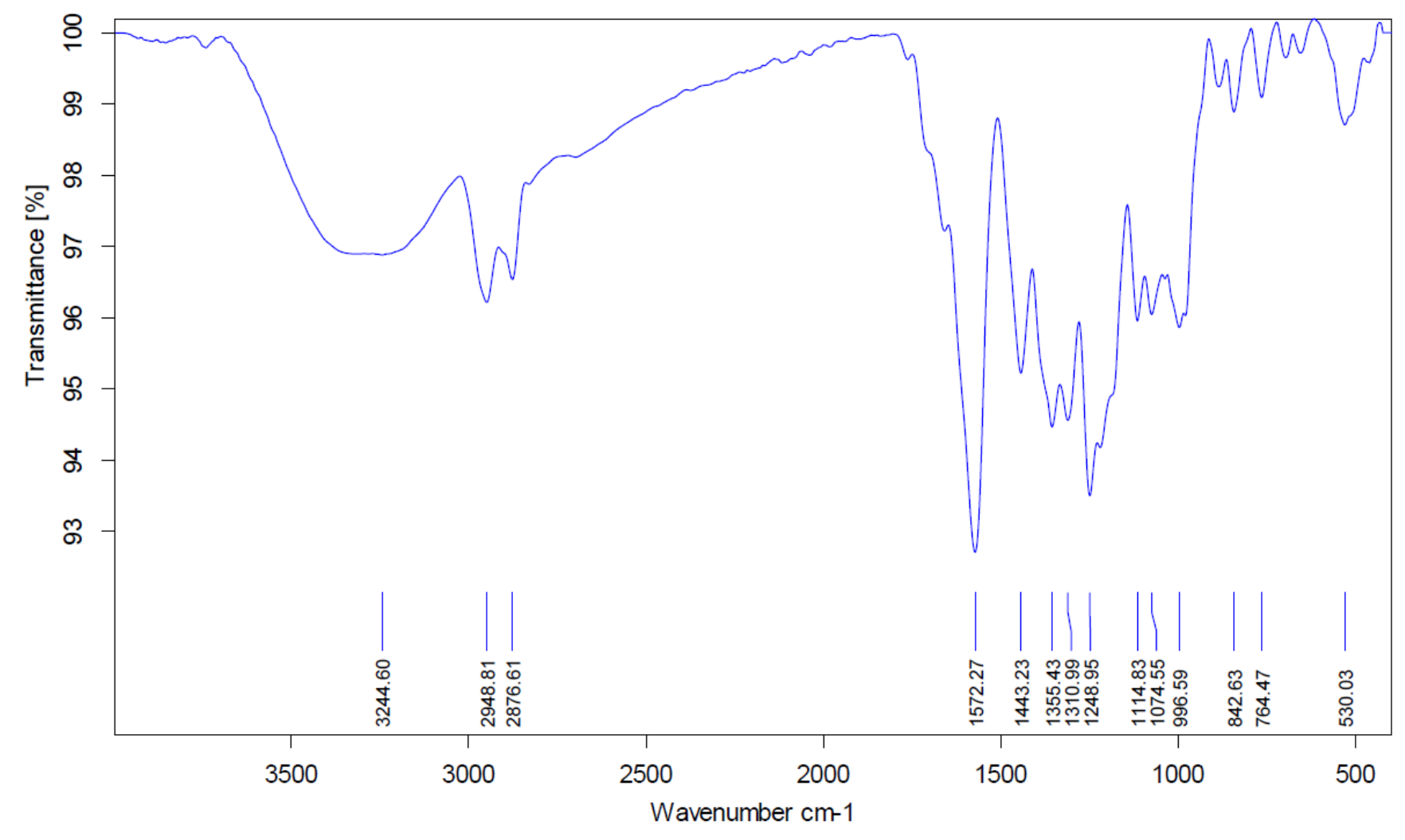

SAMPLE SCANS : 16

DATE : 06/09/2016

SAMPLE : STACHYBOTRYSIN

TIME : 04:24:20

TECHNIQUE : ATR / DTGS

USER : YILEE

Fig. S8. IR spectrum of 1 


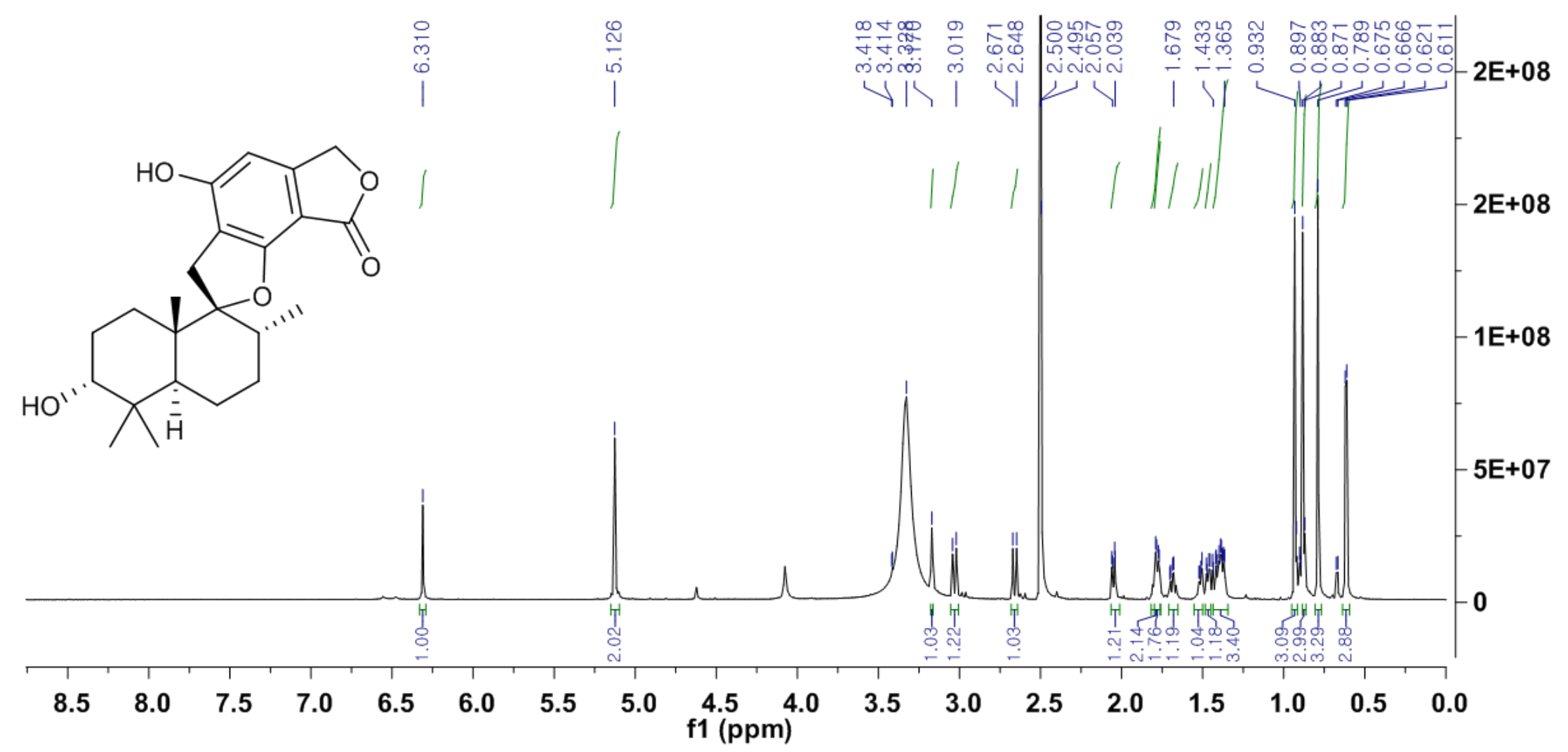

Fig. S9. ${ }^{1} \mathrm{H}$ NMR (700 MHz, DMSO-d $)$ spectrum of 2 


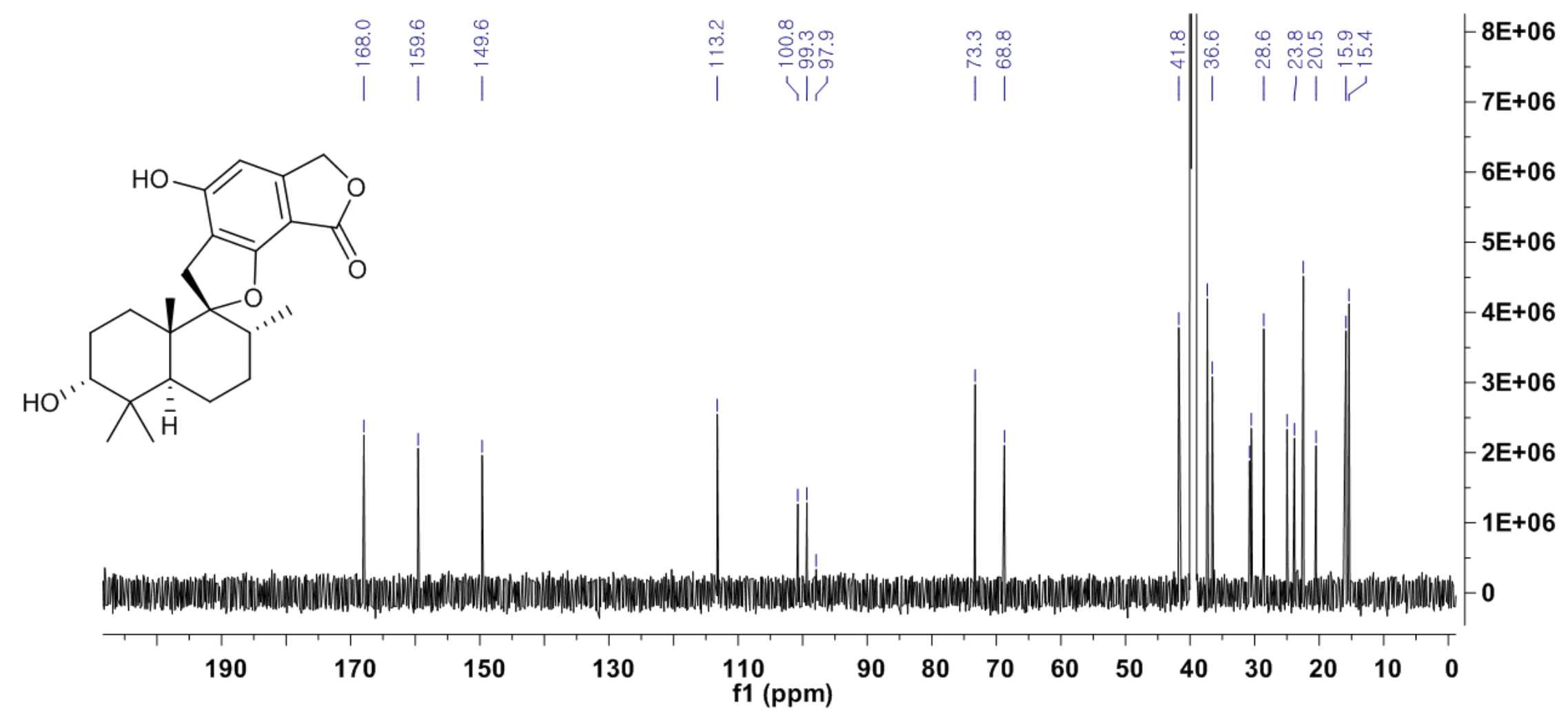

Fig. S10. ${ }^{13}$ C NMR (175 MHz, DMSO-d $)$ spectrum of 2 


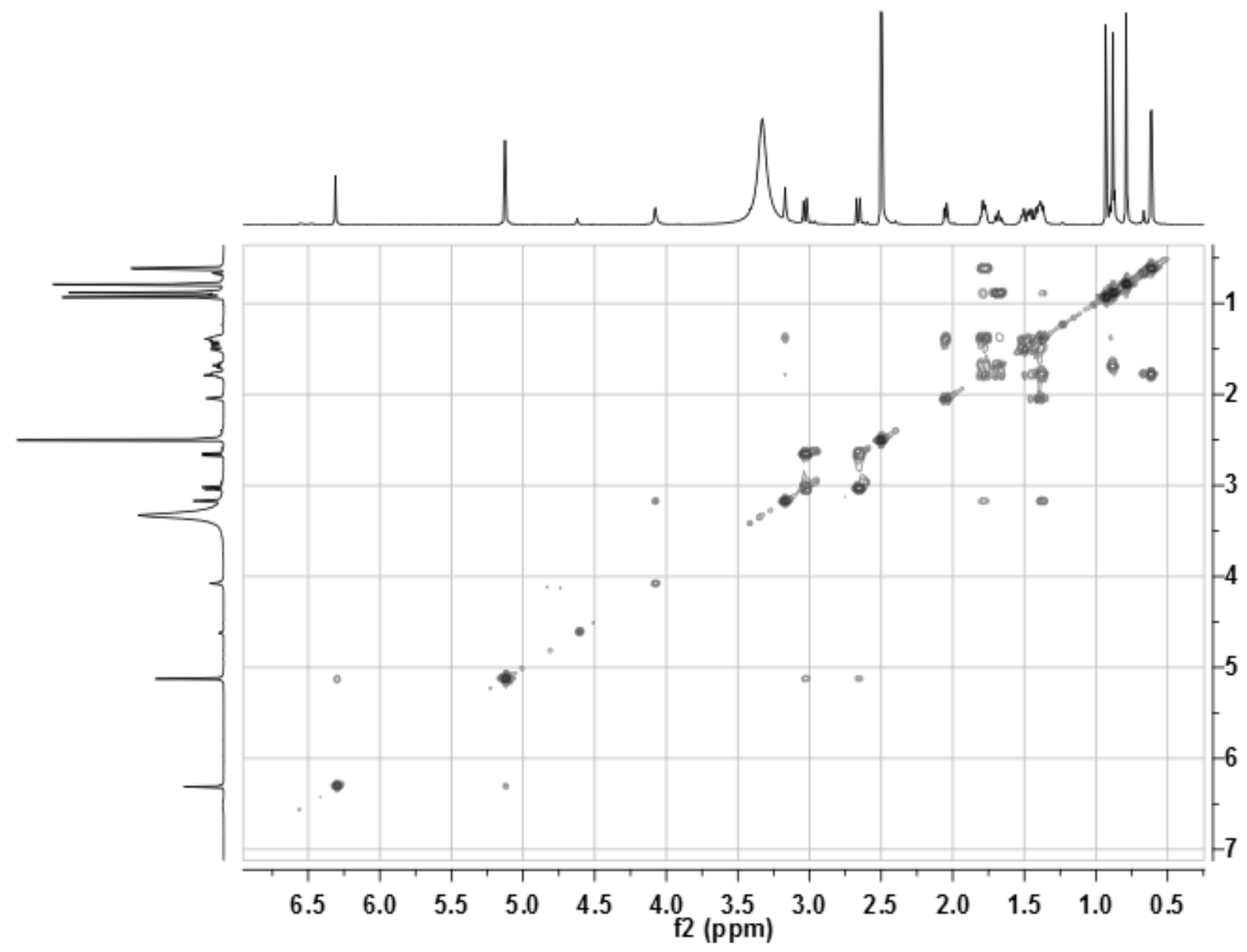

$\frac{\text { E }}{\text { 을 }}$

Fig. S11. COSY (DMSO- $d_{6}$ ) spectrum of 2 


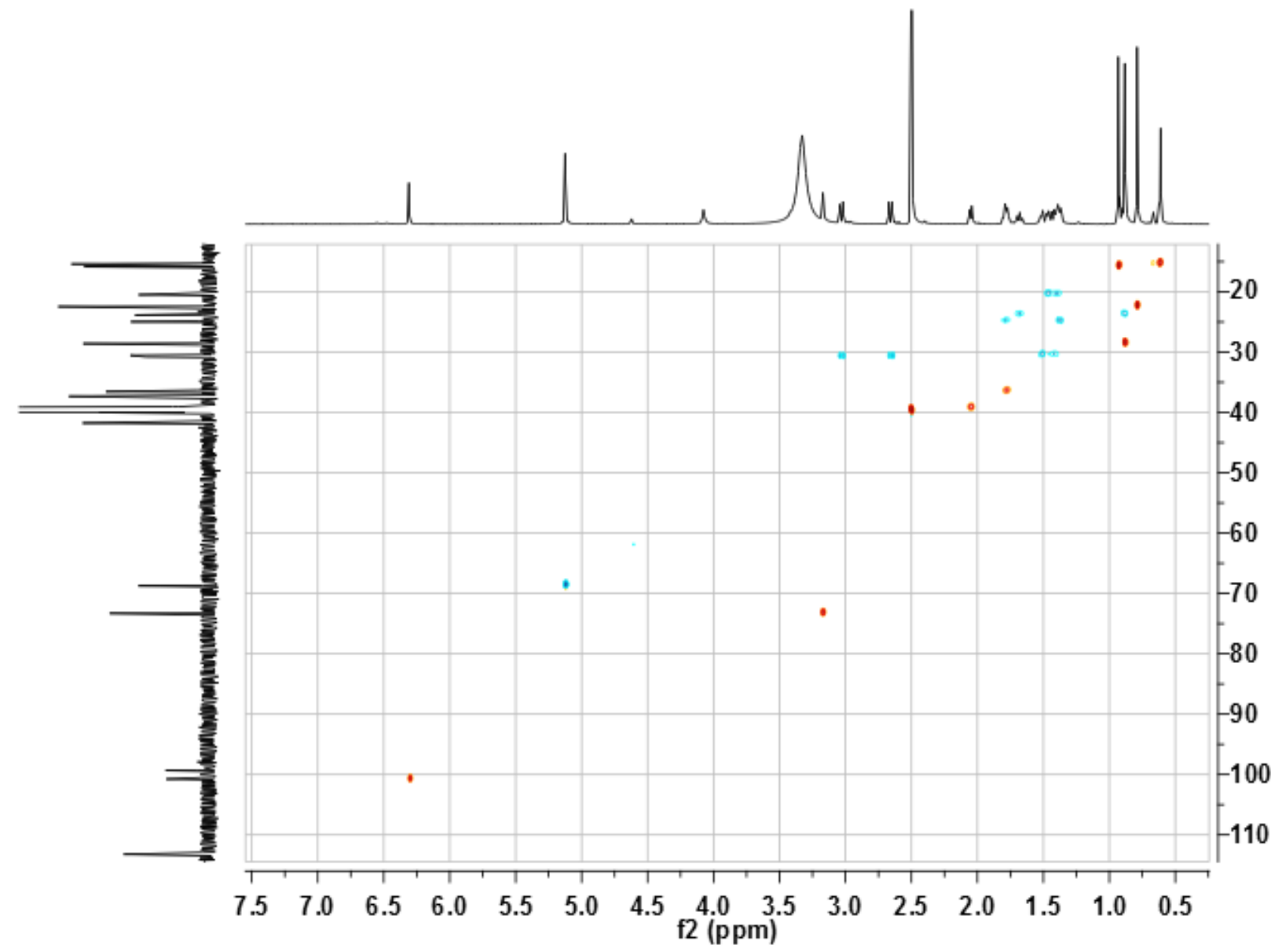

흔

Fig. S12. HSQC-DEPT (DMSO- $d_{6}$ ) spectrum of 2 


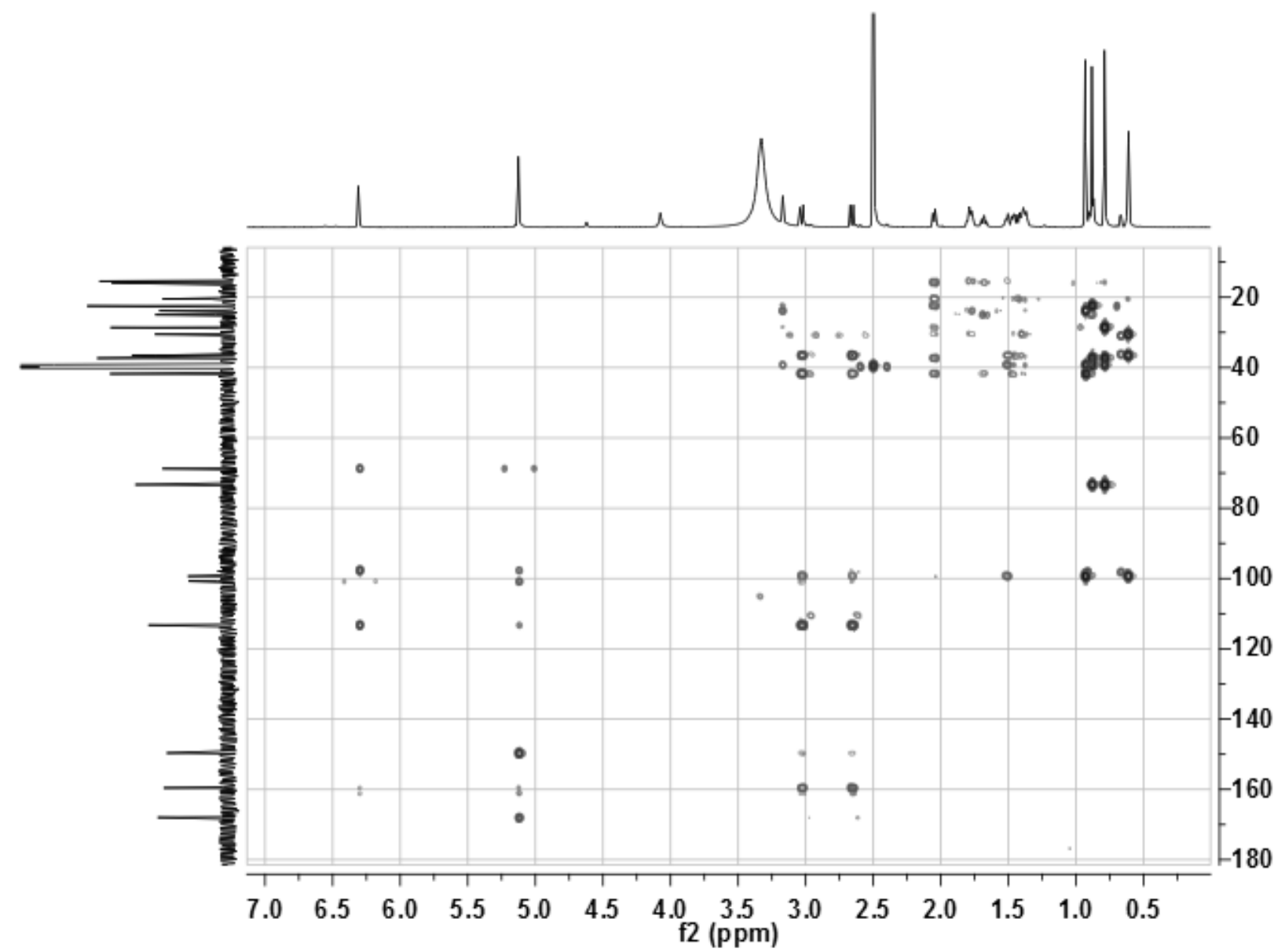

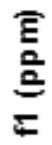

Fig. S13. HMBC (DMSO- $d_{6}$ ) spectrum of 2 


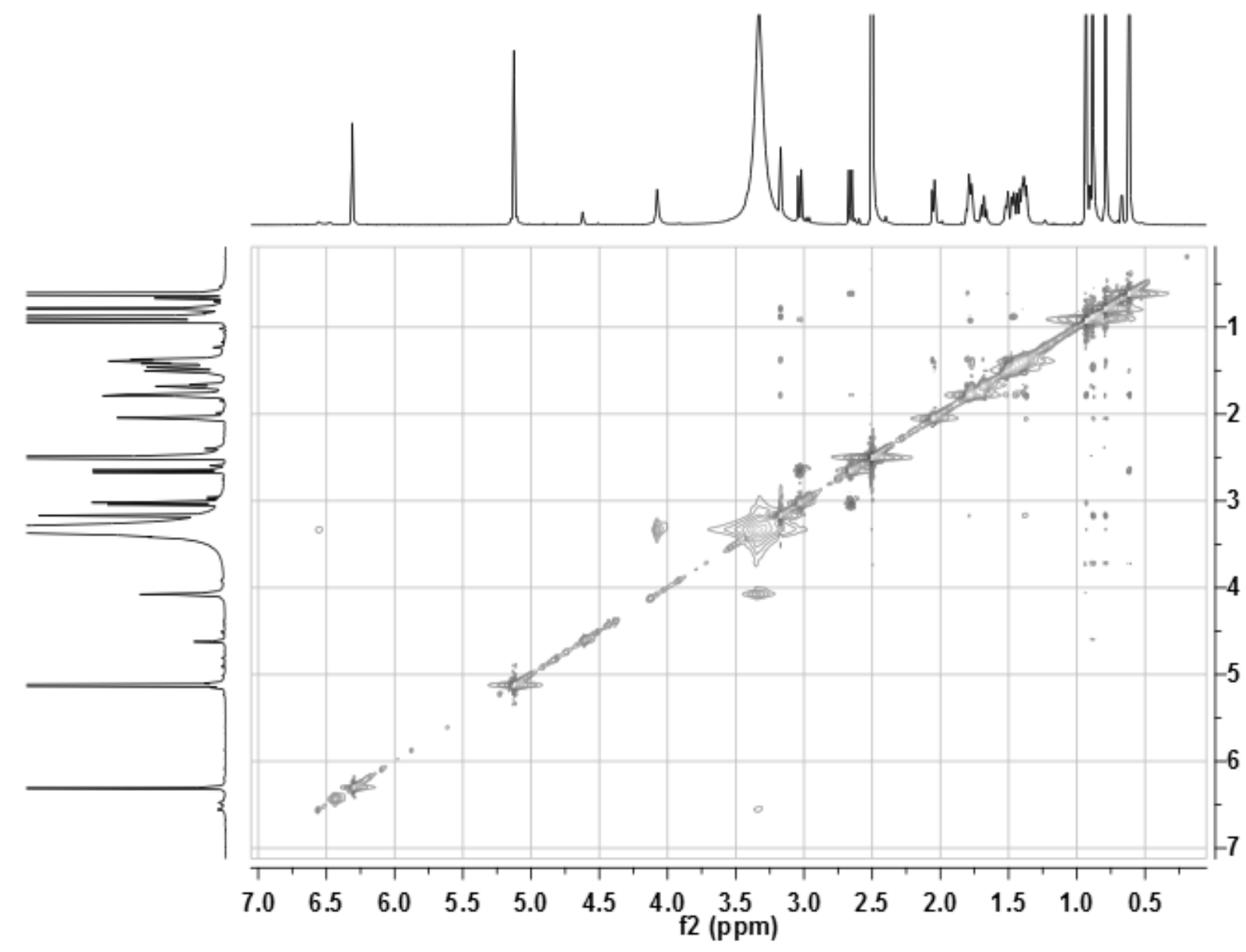

톨

Fig. S14. ROESY (DMSO-d $)$ spectrum of 2 


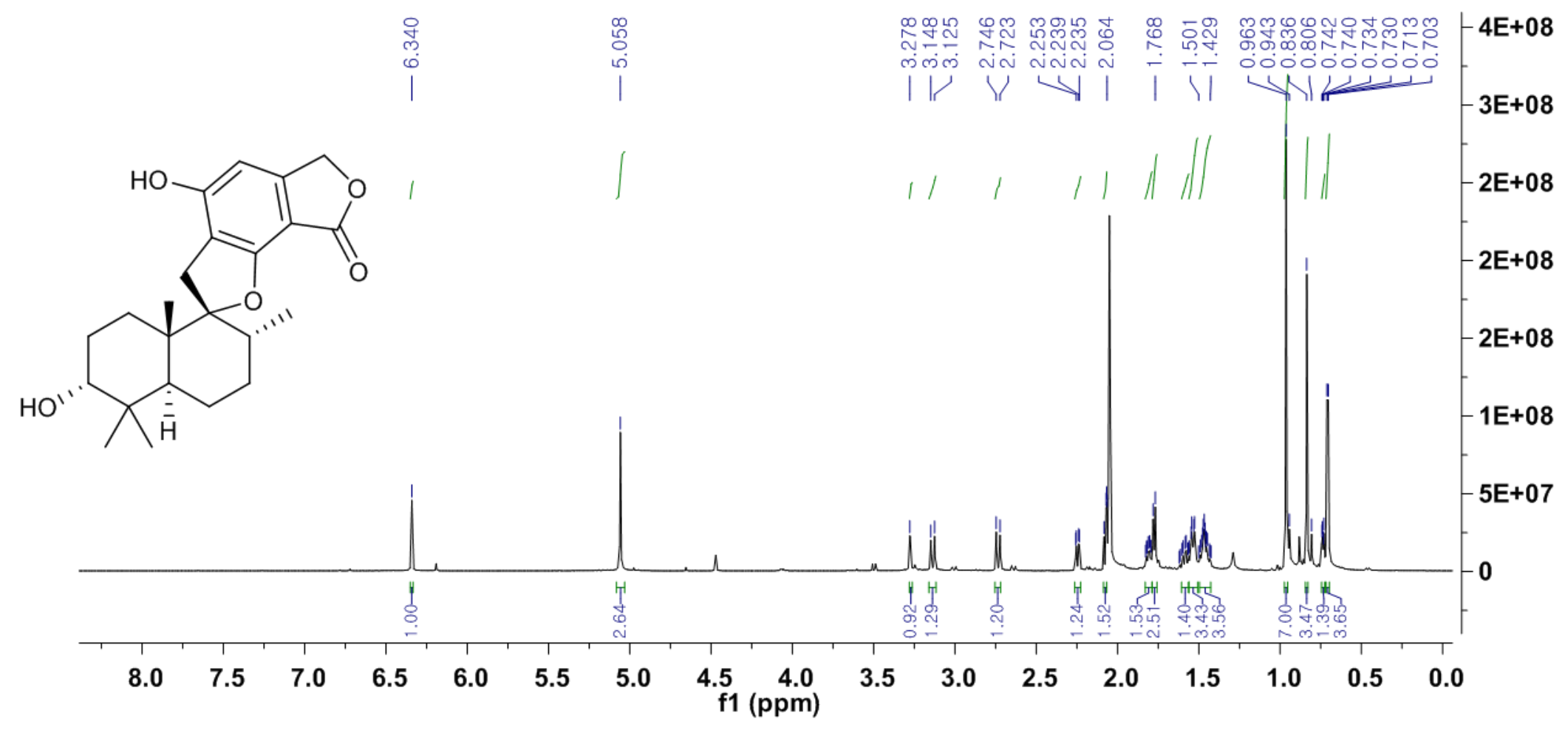

Fig. S15. ${ }^{1} \mathrm{H}$ NMR (700 MHz, Acetone- $\left.d_{6}\right)$ spectrum of 2 


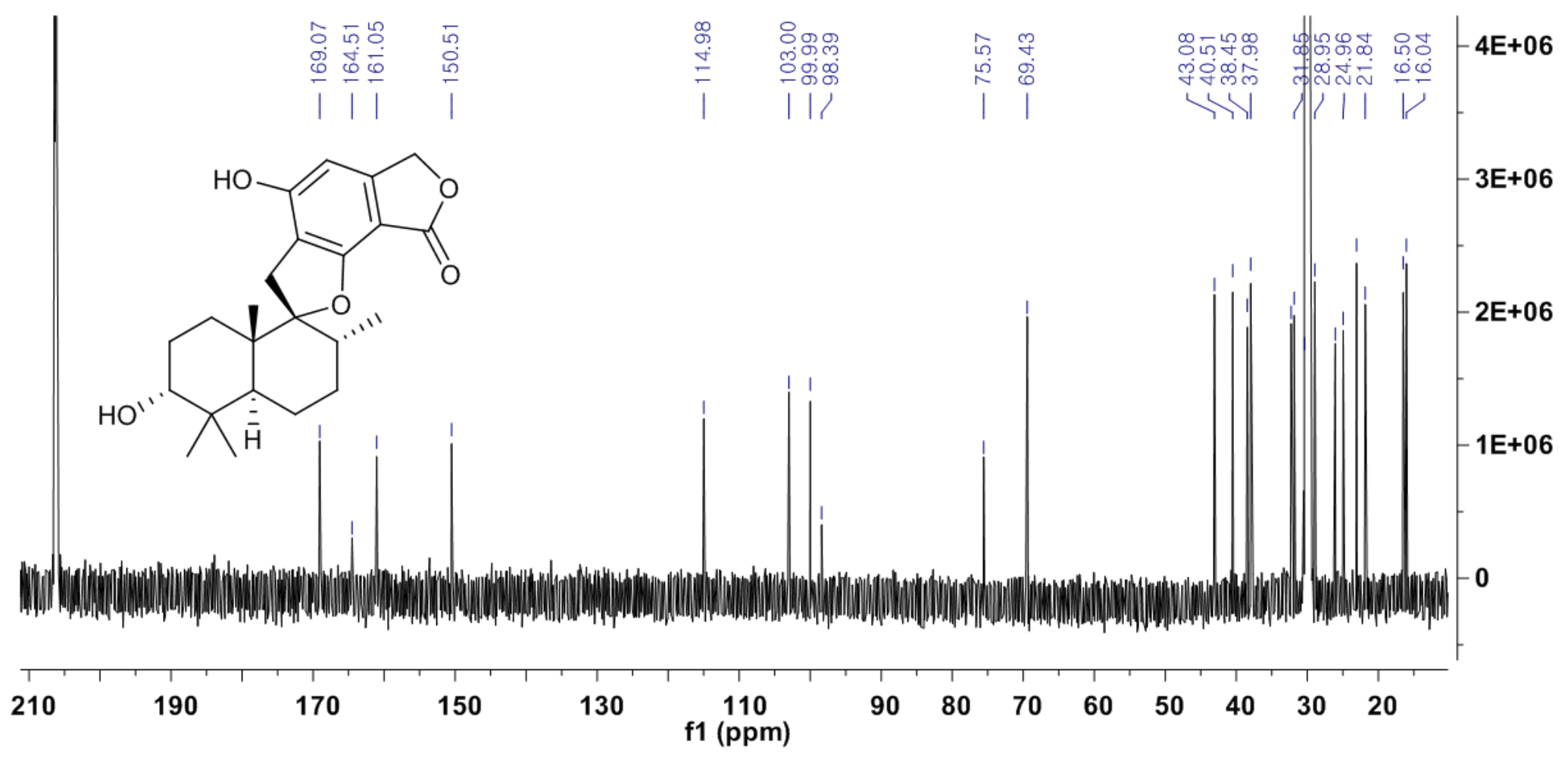

Fig. S16. ${ }^{13} \mathrm{C}$ NMR (175 MHz, Acetone-d6) spectrum of 2 


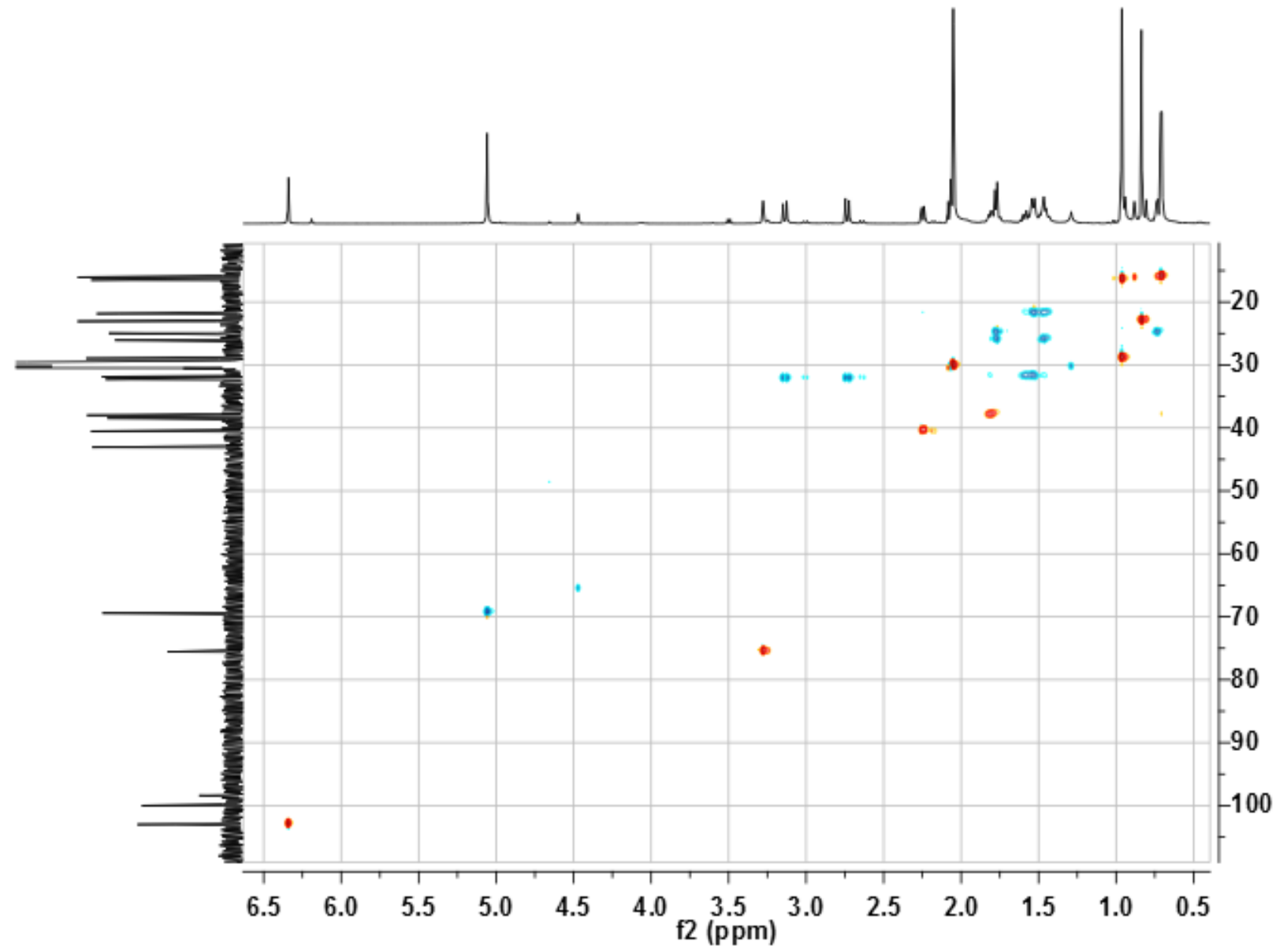

Fig. S17. HSQC-DEPT (Acetone-d6) spectrum of 2 


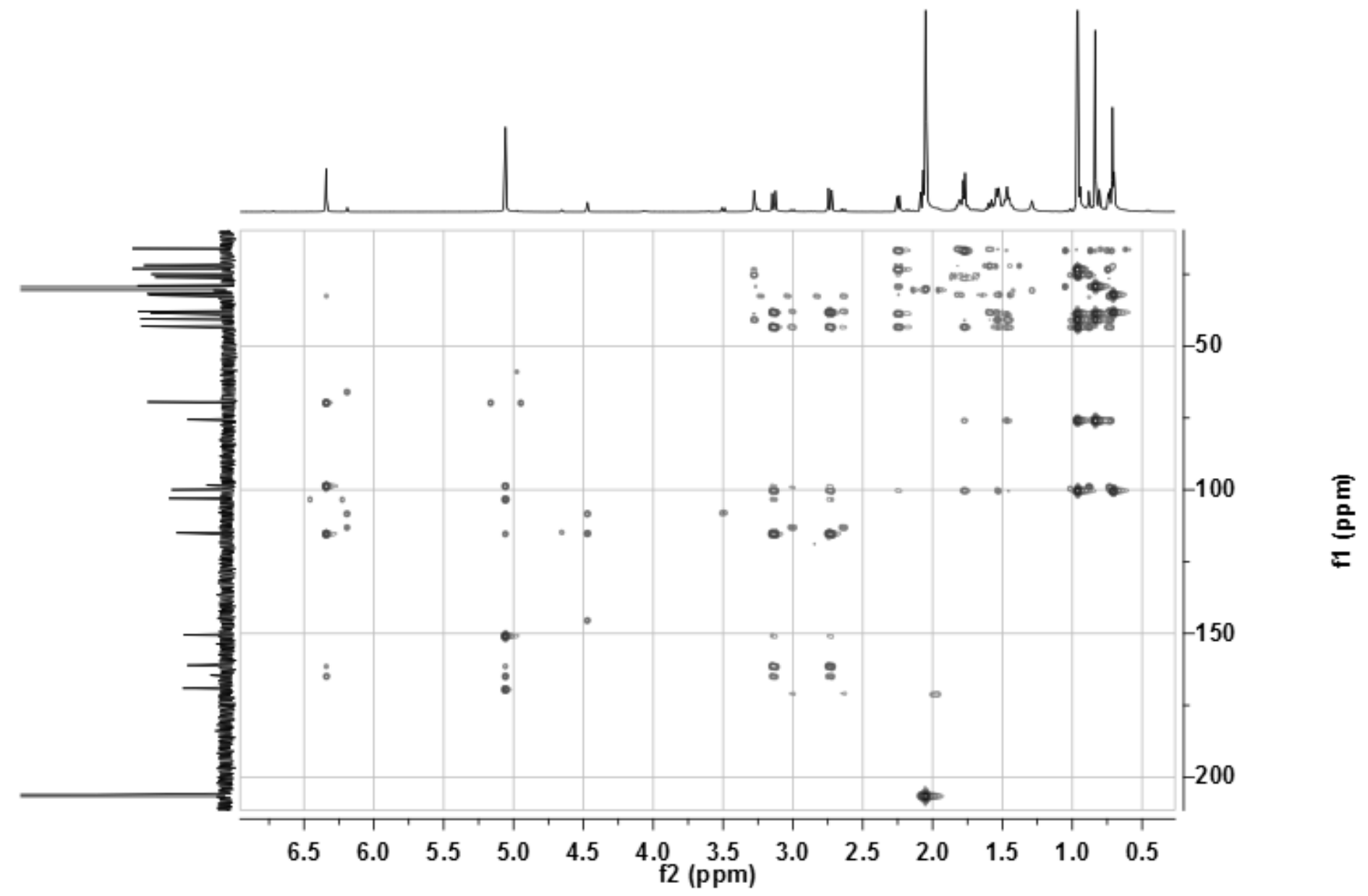

Fig. S18. HMBC (Acetone-d6) spectrum of 2 


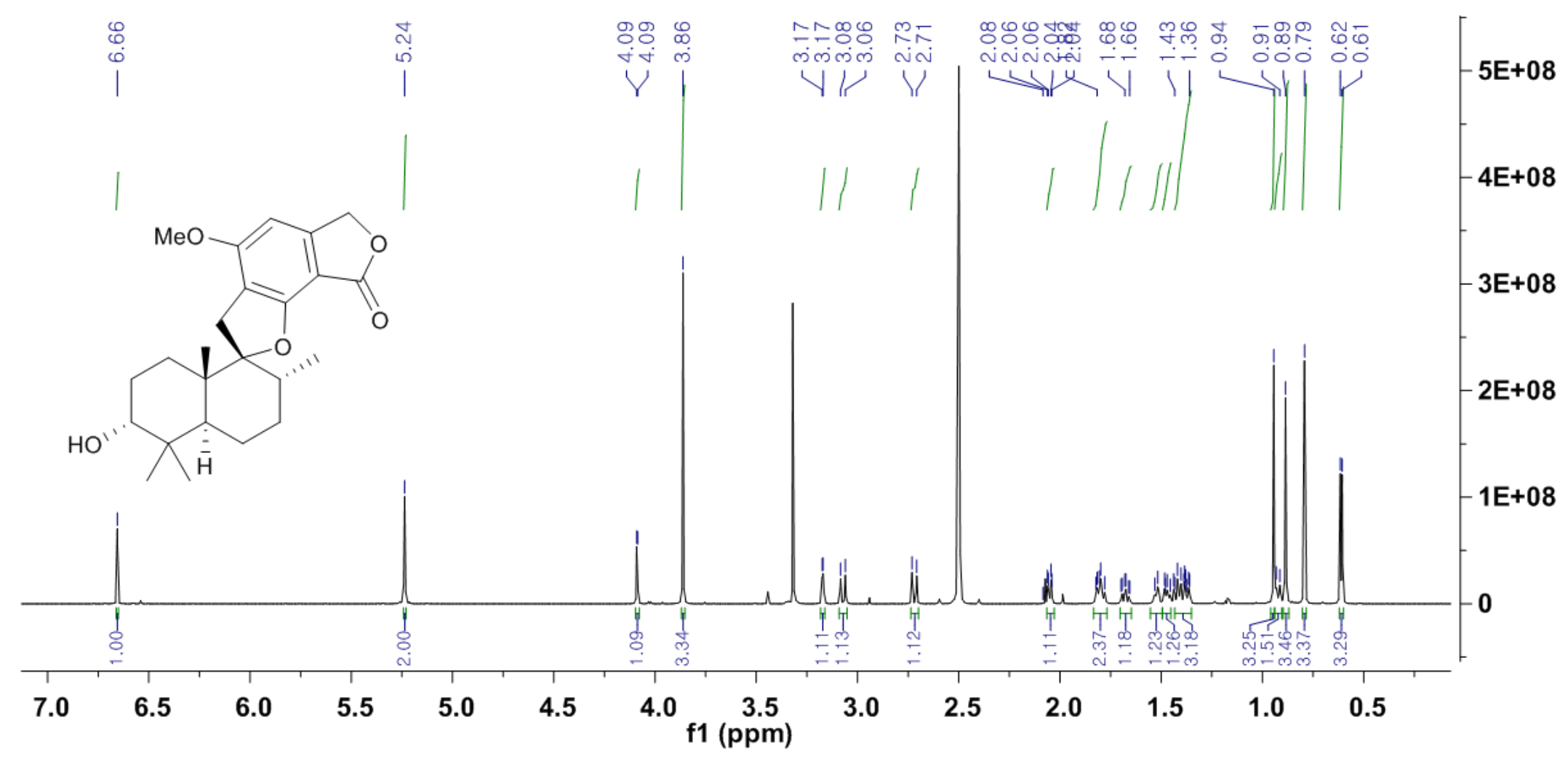

Fig. S19. ${ }^{1} \mathrm{H}$ NMR (700 MHz, DMSO- $\left.d_{6}\right)$ spectrum of methylation $2 \mathrm{~b}$ 


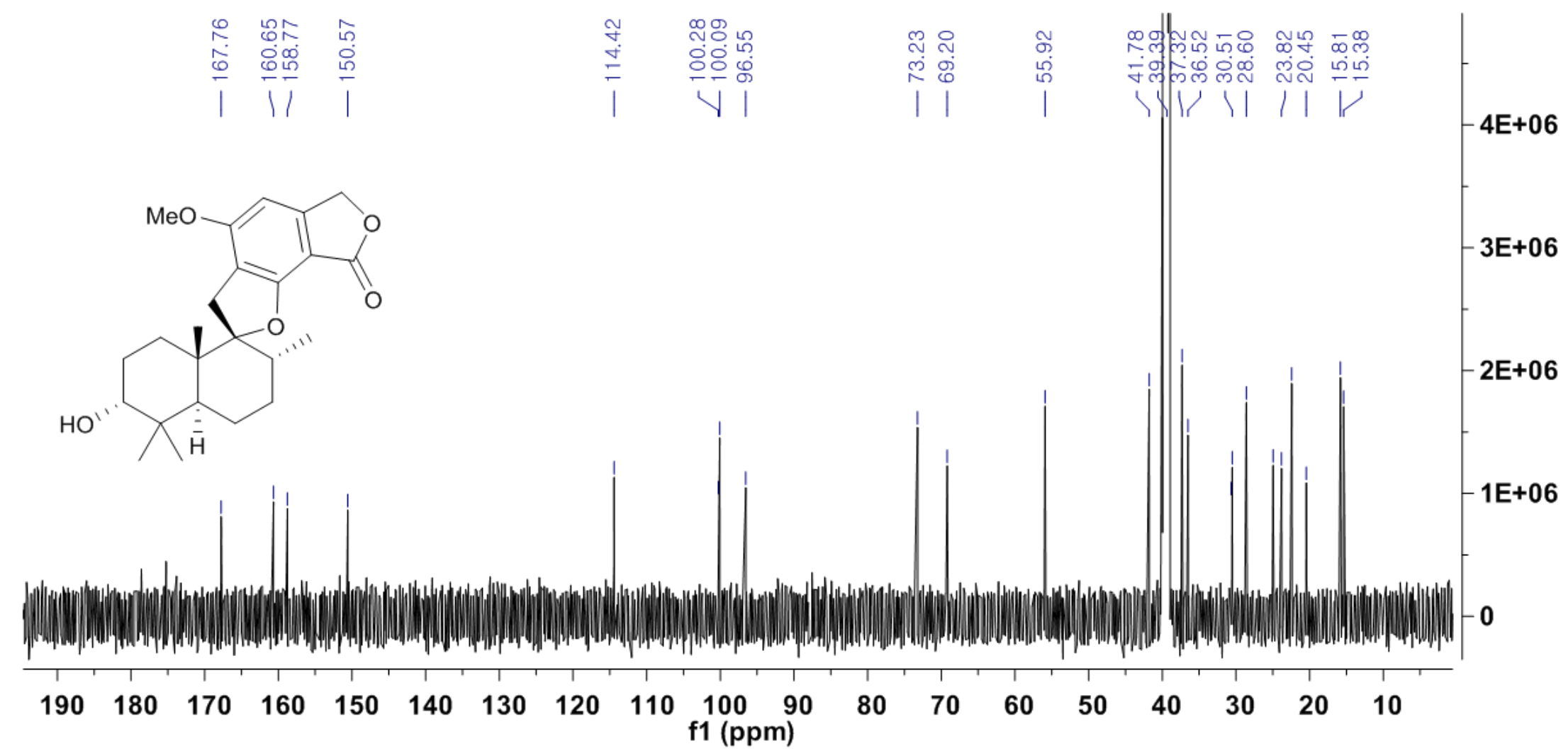

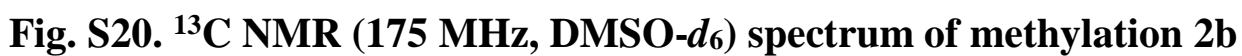




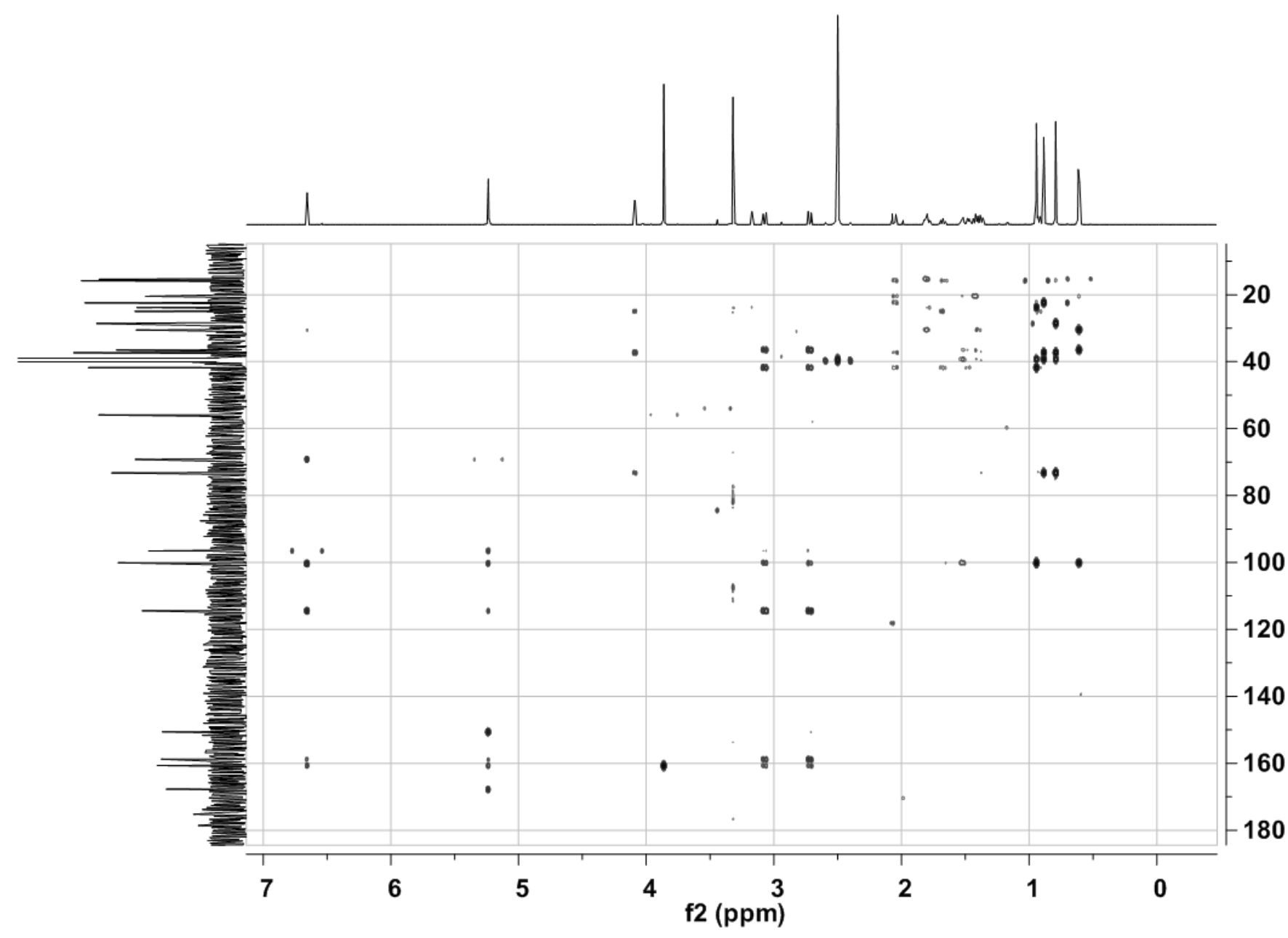

틍

Fig. S21. HMBC (DMSO-d6) spectrum of methylation 2b 


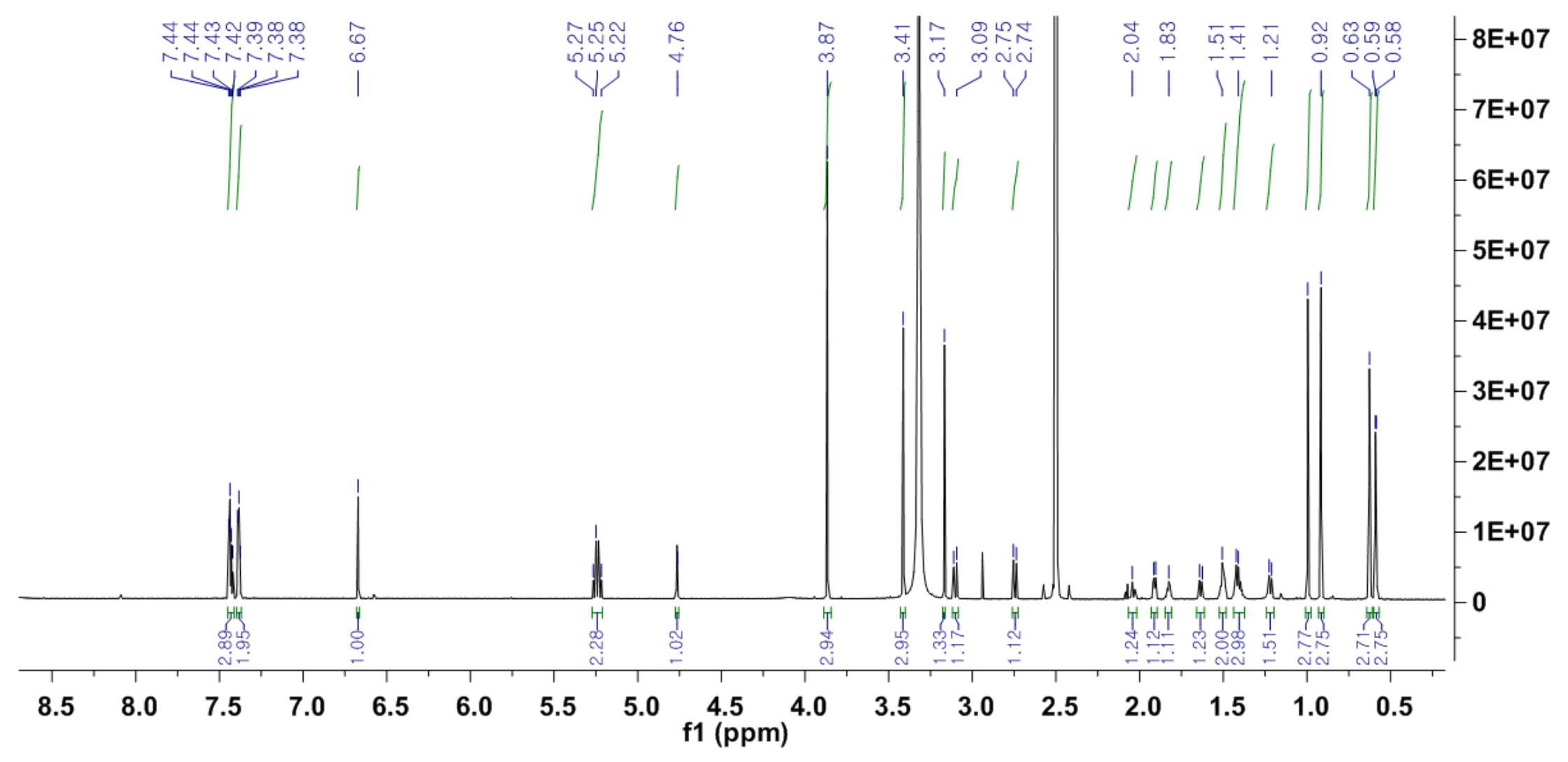

Fig. S22. ${ }^{1}$ H NMR (900 MHz, DMSO- $\left.d_{6}\right)$ spectrum of (S)-MTPA ester 2c 


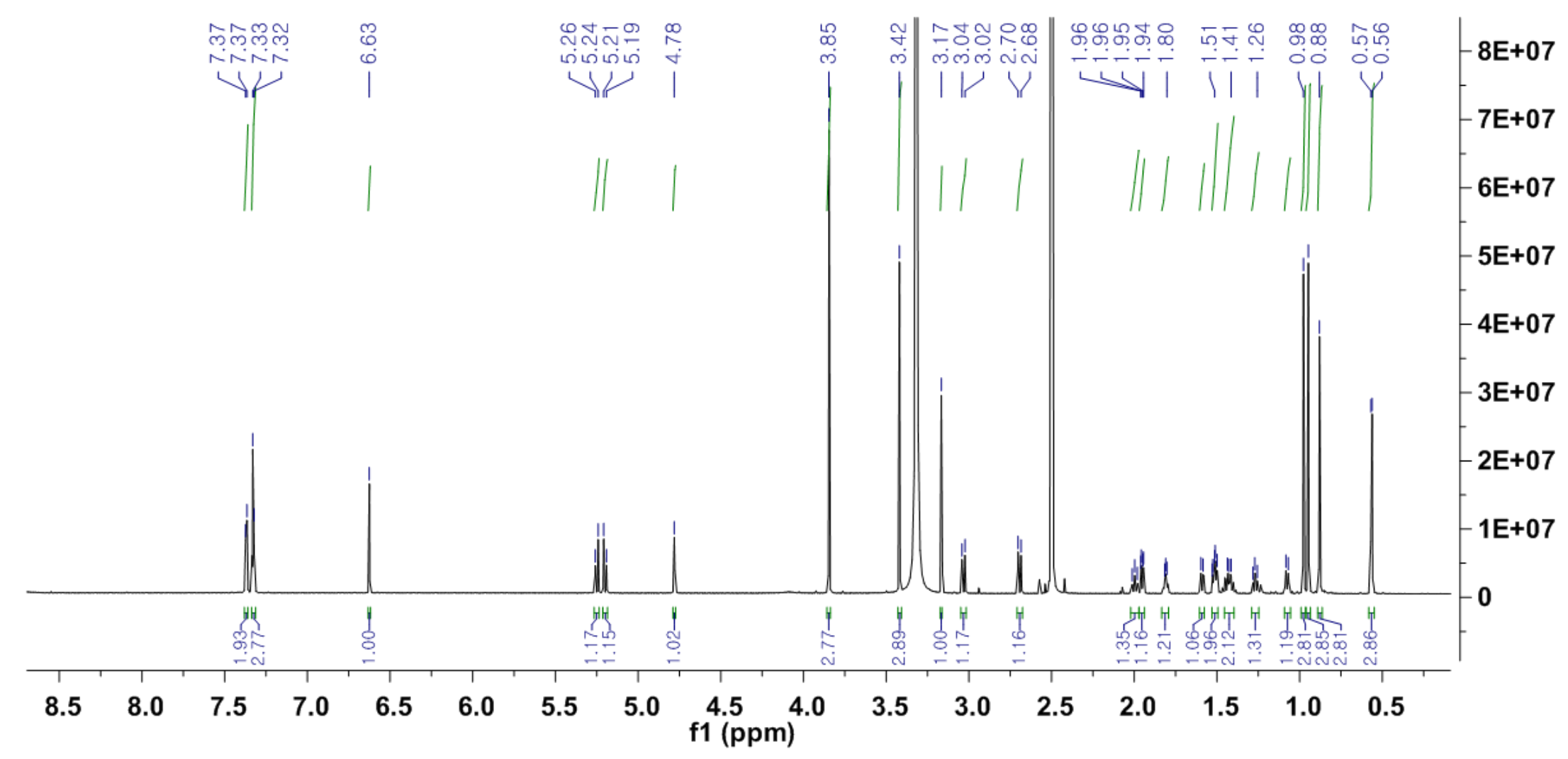

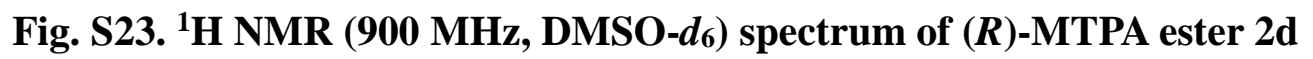


Elemental Composition Report

Single Mass Analysis

Tolerance $=5.0$ PPM / DBE: $\min =-1.5, \max =50.0$

Element prediction: Off

Number of isotope peaks used for i-FIT $=3$

Monoisotopic Mass, Even Electron lons

16 formula(e) evaluated with 1 results within limits (all results (up to 1000) for each mass)

Elements Used:

C: $1-30 \quad H: 1-40 \quad$ O: $1-5 \quad \mathrm{Na}: 1-1$

Minimum: $\quad-1.5$

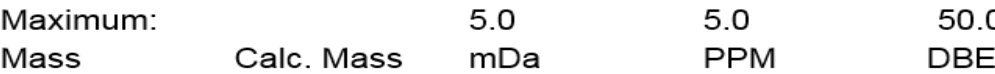

$409.1985 \quad 409.1991 \quad-0.6 \quad-1.5 \quad 8.5$

$\begin{array}{cllll}\text { DBE } & \text { i-FIT } & \text { Norm } & \text { Conf(\%) } & \text { Formula } \\ 8.5 & 948.1 & n / a & n / a & \text { C23 H30 O5 Na }\end{array}$

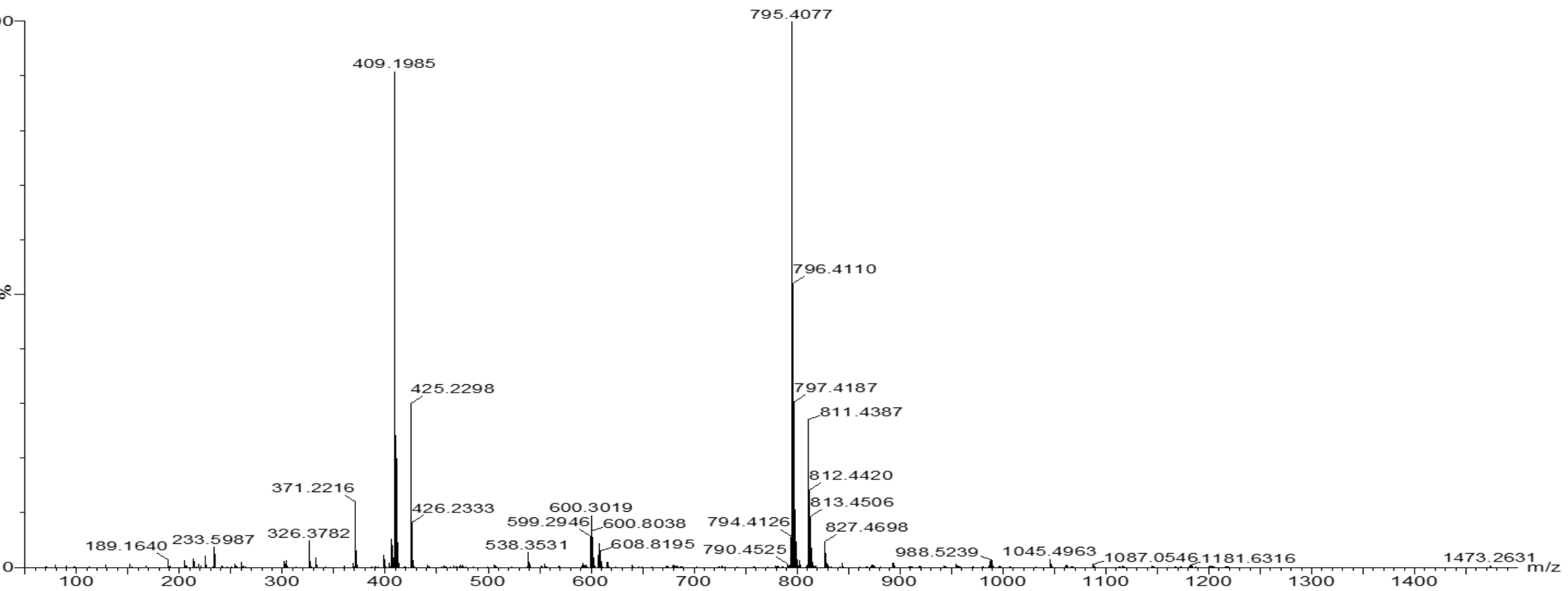

Fig. S24. Positive HRESIMS spectrum of 2 


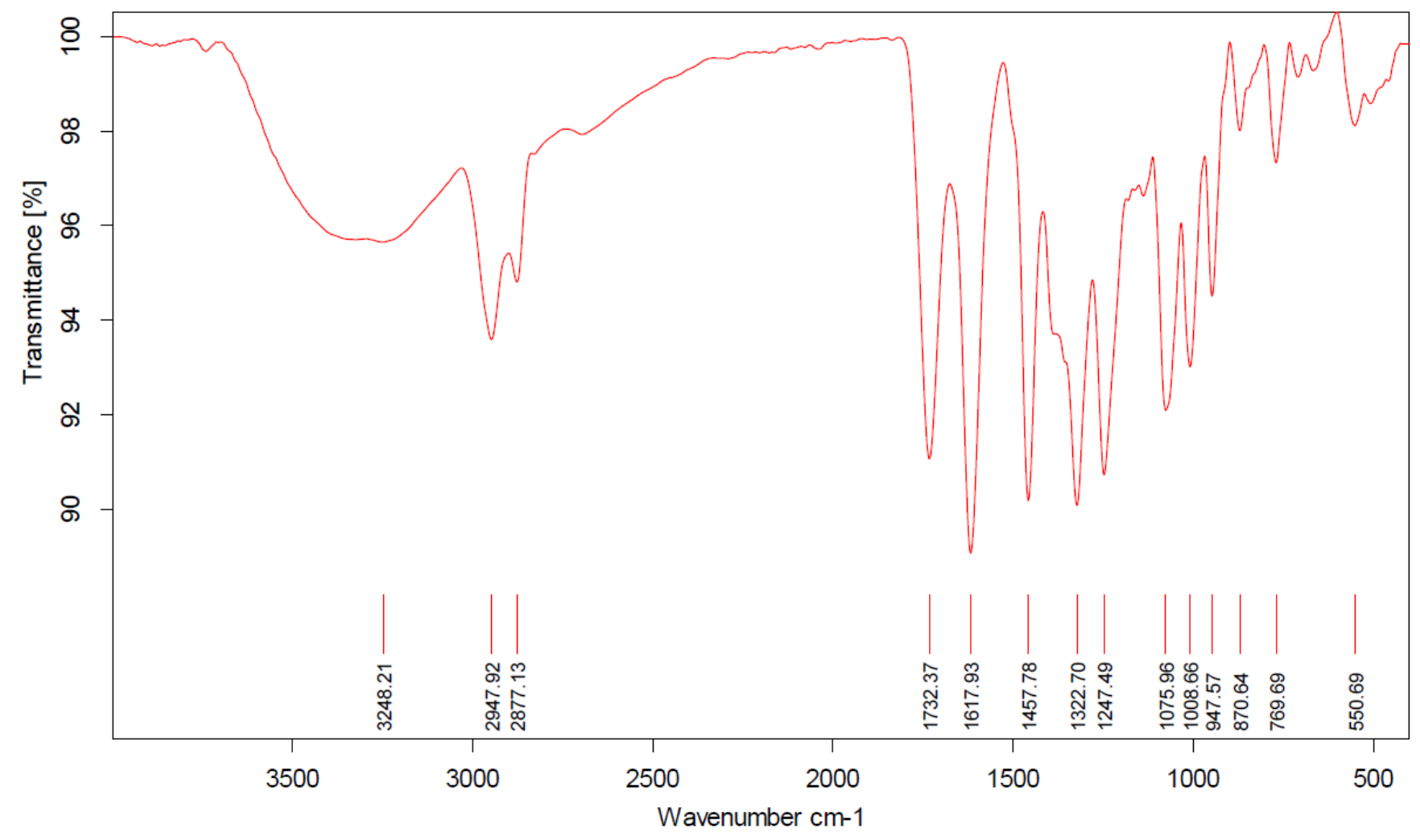

SAMPLE SCANS : 16

DATE : 06/09/2016

SAMPLE : STACHYBOTRYLACTONE B

TIME : 04:28:07

TECHNIQUE : ATR / DTGS

USER : YILEE

Fig. S25. IR spectrum of 2 
(A)

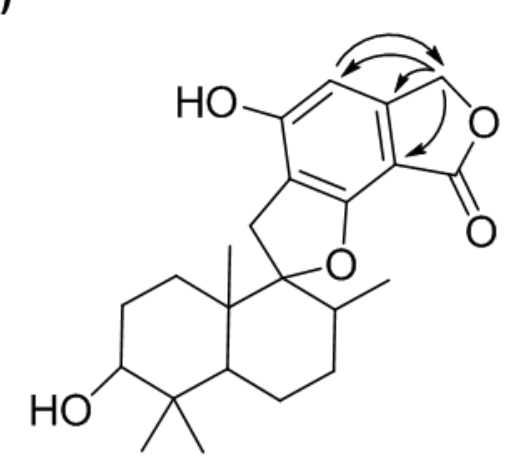

Compound 2

(B)

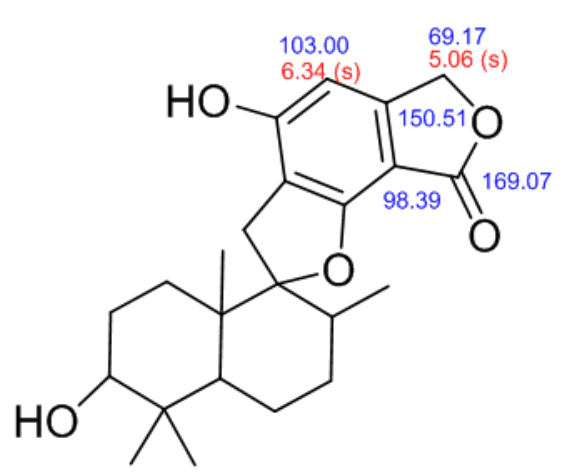

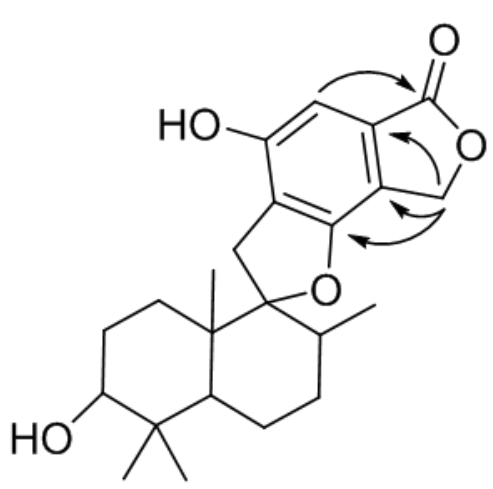

Stachybotrylactone

Ref) Can. J. Chem., 71, 487-493, 1993

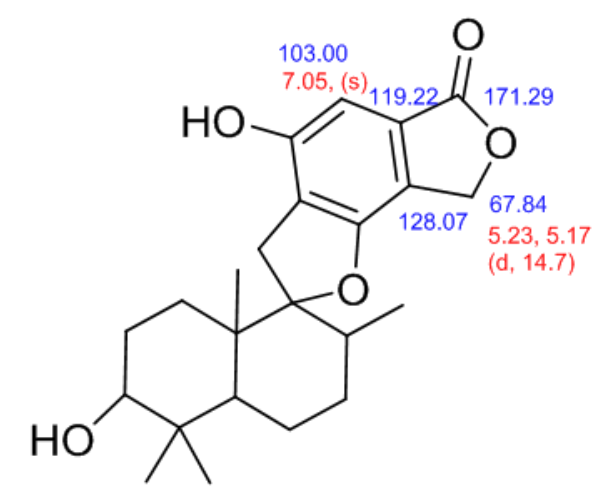

Fig. S26. The difference of HMBC correlations (A) and NMR chemical shifts (B) in acetone- $d_{6}$ 